\title{
СОЛИТОННОЕ РЕШЕНИЕ ЭЛЕКТРОМЕХАНИЧЕСКИХ ПЕРЕХОДНЫХ ПРОЦЕССОВ В ЭНЕРГОСИСТЕМАХ, СНАБЖАЮЩИХ НЕФТЯНЫЕ И ГАЗОВЫЕ МЕСТОРОЖДЕНИЯ
}

\author{
Исаев Юсуп Ниязбекович',
}

isaev_yusup@mail.ru

\author{
Колчанова Вероника Андреевна', \\ ni105@mail.ru
}

\author{
Кулешова Елена Олеговна', \\ kuleshova@tpu.ru \\ Филипас Александр Александрович', \\ filipas@tpu.ru
Национальный исследовательский Томский политехнический университет, Россия, 634050, г. Томск, пр. Ленина, 30.

Бесперебойное электроснабжение нефтяных и газовых месторождений остается важнейшей задачей мировой экономики. Одним из важнейших факторов, влияющих на отключение электроснабжения месторождений, является нарушение устойчивой работы генераторов. Устойчивая работа генераторов может быть нарушена при возникновении переходных процессов, вызванных короткими замыканиями или импульсными воздействиями на линии электропередач. При этом в электроэнергетической системе могут возникнуть уединенные волны - солитоны, характеризующиеся большой амплитудой и высокой скоростью распространения волны. В данной работе описываются причины возникновения таких волн. Приводится решение волнового уравнения электромеханической системы электроэнергетики, описывающего распространение уединенных волн. Решение рассматривается в фазовой плоскости, приводится численный пример расчета солитонного решения.

Цель: найти солитонные решения в переходных процессах электромеханических систем и объяснить причины их возникновения, дать объяснения этого физического явления, определить, какую роль это явление играет в оценке устойчивости работы генераторов и предложить мероприятия по устранению нарушения устойчивости при наличии солитонной волны.

Методы: метод фазовой плоскости, численное решение дифференциального уравнения методом Рунге- Кутта, метод пространства состояния.

Результаты. Обнаружено, что при приближении решения уравнения турбина-генератор к границе динамической устойчивости возникают солитоны - одиночные всплески величины угла генератора.

Выводы. При распространении эти волны ведут себя как частицы, что позволяет производить анализ обмена энергиями (потоками мощности) так же как анализ обмена энергиями механических частиц. При нарушении устойчивости возникают гармонические колебания, которые преобразуются в группу солитонов, распространение которых можно рассматривать как распространение частиц.

Ключевые слова:

Всплеск волны, солитон, электромеханические переходные процессы, динамическая система, фазовое пространство.

\section{Введение}

На сегодняшний день добыча нефти и газа требует мощных источников электроэнергии. Большую часть электроэнергии нефтегазодобывающие предприятия получают из распределительных сетей электроэнергетических компаний. При этом доля собственной генерации составляет около $30 \%$ от общего потребления электроэнергии на добывающих предприятиях. Потребители электрической энергии относятся к потребителям первой и второй категории питания, т. е. требуют бесперебойного питания [1, 2]. Одной из важнейших задач электроснабжения нефтегазовых предприятий является обеспечение устойчивой работы генераторов [3]. Нарушение устойчивой работы генератора может повлечь за собой его аварийное отключение, вывод из работы дорогого оборудования, простой в работе, аварии на добывающих предприятиях [4]. Решению данной задачи посвящено много работ. Одной из мало изученных причин возникновения неустойчивой работы генератора является возникновение уединенной волны - солитона [5-7].
При описании переходных процессов, происходящих в электромеханической системе, и исследовании динамической устойчивости используется нелинейное дифференциальное уравнение, описывающее агрегат турбина-генератор.

\section{$P(\delta)$}

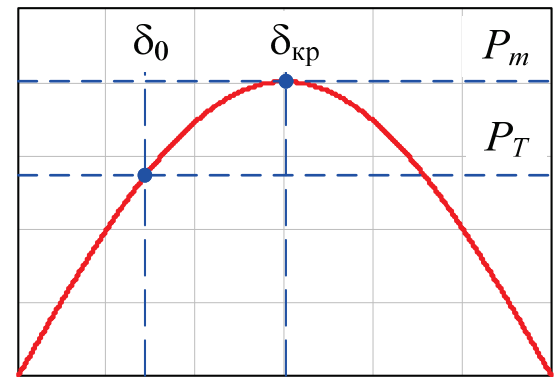

Pис.1. Угловая характеристика активной мощности генера тора, на графике показаны точка положения равновесия $\delta_{0}$ и критическая точка $\delta_{\text {кр }}$

Fig. 1. Angular characteristic of the generator active power, the graph shows the equilibrium point $\delta_{0}$ and the critical point $\delta_{\mathrm{kp}}$ 


$$
T_{J} \frac{d \omega}{d t}=P_{\grave{O}}-P_{m} \sin \left(\delta_{0}+\Delta \delta\right)
$$

или

$$
T_{J} \frac{d^{2} \delta}{d t^{2}}=P_{\grave{O}}-P_{m} \sin \left(\delta_{0}+\Delta \delta\right) .
$$

Здесь $T_{J}$ - механическая постоянная генератора; $\Delta \omega$ - отклонение частоты энергосистемы от положения равновесия; $P_{T}-$ активная мощность турбины (рис. 1$) ; P_{m} \sin (\delta)$ - активная мощность генератора. Это уравнение описывает колебания угла ротора генератора $\delta$. Отклонение угла $\Delta \delta$ от его положения равновесия $\delta_{0}$. При соблюдении баланса генерации и потребления энергии в системе, т. е. при равенстве активных мощностей турбины и генератора, отклонения частоты от положения равновесия не происходит (точка на графике $\delta_{0}-$ устойчивая точка положения равновесия) и система работает при постоянной частоте. Возмущения, вызывающие существенные отклонения угла от устойчивой работы системы, могут быть вызваны как короткими замыканиями, так и другими типами аварий. При исследовании влияния генераторов друг на друга необходимо принимать во внимание распределенность системы, т. е. учитывать зависимость угла каждого генератора не только от времени $t$, но и от координаты $x$ $[8,9]$. В таком случае следует говорить не о колебательных процессах, а о волновых процессах.

Нелинейные волновые процессы, происходящие в электромеханических системах, имеют общность с волновыми процессами, происходящими в других областях, таких как гидродинамика $[10,11]$, учитывающая распространение волн в сплошных средах [12]. В частности, известно, что в ряде случаев на гребнях волны появляются осцилляции в виде всплесков, уединенных волн, называемых солитонами (solitary wave) $[13,14]$. Решение нелинейных задач на основе солитонов является актуальным, что подтверждается работами многих авторов [15, 16]. Особенности солитонов заключаются в том, что при взаимодействии двух солитонных волн одинаковой амплитуды они напоминают процесс взаимодействия двух частиц одинаковой массы (частицеподобная волна), при котором частицы обмениваются скоростями распространения. При взаимодействии друг с другом или с некоторыми другими возмущениями солитоны не разрушаются, а продолжают движение, сохраняя свою структуру неизменной $[13,17]$.

В данной работе авторами предпринята попытка найти солитоны в переходных процессах электромеханических систем, объяснить причины их возникновения и дать объяснения этого физического явления, определить, какую роль это явление играет в оценке устойчивости и предложить мероприятия по устранению нарушения устойчивости при наличии солитонной волны.

Ограничимся рассмотрением стационарного случая, когда волновое уравнение в частных производных преобразуется в обыкновенное дифференциальное уравнение типа (1) для уединенной энергосистемы $[13,18]$. Анализ динамической системы будем производить с помощью фазовой плоскости $\delta, \omega$.

Перепишем уравнение (1) в виде системы уравнений первого порядка

$$
\left\{\begin{array} { l } 
{ \frac { d \delta } { d t } = \omega ; } \\
{ \frac { d \omega } { d t } = \frac { P _ { \grave { o } } - P _ { m } \operatorname { s i n } ( \delta ) } { T _ { J } } . }
\end{array} \text { или } \left\{\begin{array}{l}
\frac{d \delta}{d t}=f_{1}(\omega, \delta) ; \\
\frac{d \omega}{d t}=f_{2}(\omega, \delta) .
\end{array}\right.\right.
$$

Опишем последовательность действий исследуемой системы:

1. Находим точки положения равновесия на фазовой плоскости, прировняв все производные нулю (установившийся процесс). Число корней может быть больше, чем порядок дифференциального уравнения, в отличие от линейной системы, где число корней строго соответствует порядку уравнений. В нашем случае мы имеем бесконечное количество корней вдоль оси углов, поэтому ограничимся рассмотрением корней уравнения (2) в первом полупериоде:

$$
\left\{\begin{array}{l}
0=f_{1}(\omega, \delta) \\
0=f_{2}(\omega, \delta)
\end{array} \rightarrow \omega=\omega_{0}, \delta=\delta_{0}, \omega=\omega_{0}, \delta=\delta_{1}\right. \text {. }
$$

2. Раскладываем функции $f_{1}(\omega, \delta)$ и $f_{2}(\omega, \delta)$ в окрестности точки положения равновесия $\omega=\omega_{0}, \delta=\delta_{0}:$

$$
\left\{\begin{array}{l}
f_{1}(\omega, \delta)=f_{1}\left(\omega_{0}, \delta_{0}\right)+\left.\frac{\partial f_{1}}{\partial \delta}\right|_{\delta=\delta_{0}} \Delta \delta+\left.\frac{\partial f_{1}}{\partial \omega}\right|_{\omega=\omega_{0}} \Delta \omega+\ldots \\
f_{2}(\omega, \delta)=f_{2}\left(\omega_{0}, \delta_{0}\right)+\left.\frac{\partial f_{2}}{\partial \delta}\right|_{\delta=\delta_{0}} \Delta \delta+\left.\frac{\partial f_{2}}{\partial \omega}\right|_{\omega=\omega_{0}} \Delta \omega+\ldots
\end{array}\right.
$$

где

$$
\Delta \delta=\delta-\delta_{0}, \quad \Delta \omega=\omega-\omega_{0} .
$$

Аналогичные действия производим и для второй точки положения равновесия $\omega=\omega_{0}, \delta=\delta_{1}$.

3. Формируем матрицу Якоби, выписывая коэффициенты при линейных членах:

$$
\begin{gathered}
\mathbf{A}(\delta, \omega)=\left(\begin{array}{ll}
\frac{\partial f_{1}}{\partial \delta} & \frac{\partial f_{1}}{\partial \omega} \\
\frac{\partial f_{2}}{\partial \delta} & \frac{\partial f_{2}}{\partial \omega}
\end{array}\right), \\
\mathbf{A}_{1}=\mathbf{A}\left(\delta_{0}, \omega_{0}\right), \quad \mathbf{A}_{2}=\mathbf{A}\left(\delta_{1}, \omega_{0}\right) .
\end{gathered}
$$

Собственные числа матриц Якоби (4) и (5) позволят нам определить характер точек положения равновесия (3)

$$
\begin{aligned}
& \left\{\begin{array}{l}
\frac{d \Delta \delta}{d t}=\Delta \omega ; \\
\frac{d \Delta \omega}{d t}=-\frac{\Delta P}{T_{J}}=-\frac{1}{T_{J}}\left(\frac{\partial P}{\partial \delta} \Delta \delta+\frac{\partial P}{\partial \omega} \Delta \omega\right) .
\end{array}\right. \\
& \rightarrow\left(\begin{array}{c}
\frac{d \Delta \delta}{d t} \\
\frac{d \Delta \omega}{d t}
\end{array}\right)=\left(\begin{array}{cc}
0 & 1 \\
-\frac{1}{T_{J}} \frac{\partial P}{\partial \delta} & -\frac{1}{T_{J}} \frac{\partial P}{\partial \omega}
\end{array}\right)\left(\begin{array}{l}
\Delta \delta \\
\Delta \omega
\end{array}\right) .
\end{aligned}
$$




$$
\begin{gathered}
\left(\begin{array}{c}
\frac{d \Delta \delta}{d t} \\
\frac{d \Delta \omega}{d t}
\end{array}\right)=\mathbf{A}\left(\begin{array}{c}
\Delta \delta \\
\Delta \omega
\end{array}\right) \rightarrow \\
\rightarrow \mathbf{A}=\left(\begin{array}{cc}
0 & 1 \\
-\frac{1}{T_{J}} \frac{\partial P}{\partial \delta} & -\frac{1}{T_{J}} \frac{\partial P}{\partial \omega}
\end{array}\right)=\left(\begin{array}{cc}
0 & 1 \\
-\frac{\mathrm{P}_{m} \cos (\delta)}{T_{J}} & 0
\end{array}\right) .
\end{gathered}
$$

В интересующей нас области имеются две точки положения равновесия, тогда из (6)

$$
\mathbf{A}\left(\delta_{0}, \omega_{0}\right)=\mathbf{A}_{1}=\left.\left(\begin{array}{cc}
0 & 1 \\
-\frac{\mathrm{P}_{m} \cos (\delta)}{T_{J}} & 0
\end{array}\right)\right|_{\substack{\omega=0 \\
\delta=\delta_{0}}}
$$

и

$$
\mathbf{A}\left(\delta_{1}, \omega_{0}\right)=\mathbf{A}_{2}=\left.\left(\begin{array}{cc}
0 & 1 \\
-\frac{\mathrm{P}_{m} \cos (\delta)}{T_{J}} & 0
\end{array}\right)\right|_{\substack{\omega=0=\delta_{1} \\
\delta=\delta_{1}}}
$$

Записываем характеристическое уравнение в случае первой точки равновесия (7)

$$
p^{2}+\frac{1}{T_{J}} \frac{\partial P}{\partial \delta}=0 \rightarrow p= \pm j \sqrt{\frac{1}{T_{J}} \frac{\partial P}{\partial \delta}}= \pm j \omega .
$$

Из (8) видно, что тип точки положения равновесия - иентр (рис. 2).

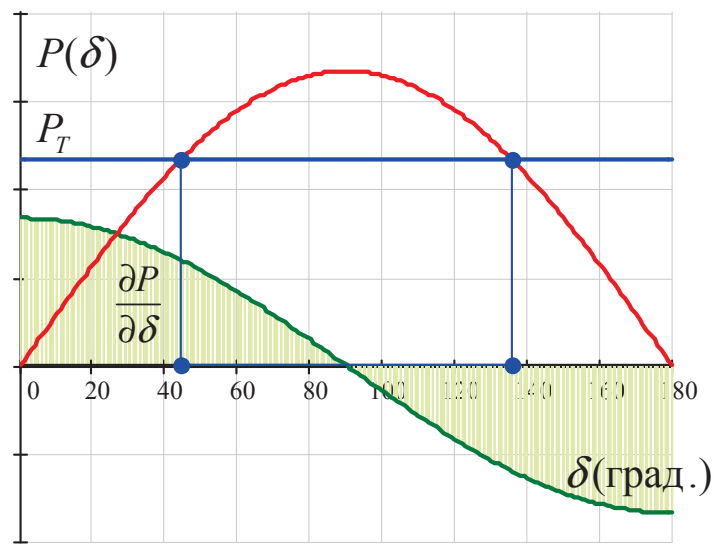

Puс. 2. Угловая характеристика активной мощности $P(\delta)$ од номашинной системы и график синхронизирующей мошности $\partial P / \partial \delta$. Кружками указаны точки положения рав новесия

Fig. 2. Angular characteristic of active power $P(\delta)$ of the single machine system and the graph of synchronizing power $\partial P / \partial \delta$. Circles indicate equilibrium points

Записываем характеристическое уравнение в случае второй точки равновесия (7)

$$
p^{2}-\frac{1}{T_{J}}\left|\frac{\partial P}{\partial \delta}\right|=0 \rightarrow p= \pm \sqrt{\frac{1}{T_{J}} \frac{\partial P}{\partial \delta}}= \pm \alpha_{1,2} .
$$

Из (9) видно, что тип точки положения равновесия - седло.

Вернемся к уравнению относительного движения ротора генератора (1)

$$
T_{J} \frac{d \omega}{d t}=P-P_{m} \sin (\delta)=\Delta P .
$$

Запишем выражение (10) в энергетическом представлении, сделав ряд преобразований

$$
\begin{aligned}
T_{J} \frac{d \omega}{d \delta} \frac{d \delta}{d t} & =\Delta P \rightarrow T_{J} \omega \frac{d \omega}{d \delta}=\Delta P \rightarrow \\
& \rightarrow \frac{d}{d \delta} \frac{T_{J} \omega^{2}}{2}=\Delta P .
\end{aligned}
$$

Проинтегрируем выражение (11) по углу и получим интеграл энергии

$$
\int \frac{d}{d \delta} \frac{T_{J} \omega^{2}}{2} d \delta=\int \Delta P d \delta \rightarrow \frac{T_{J} \omega^{2}}{2}-\int \Delta P d \delta=C .
$$

Выражение (12) - это уравнение сохранения энергии

$$
\frac{T_{J} \omega^{2}}{2}+U(\delta)=E .
$$

Рассмотрим выражение для потенциальной энергии

$$
\begin{gathered}
U(\delta)=-\int_{0}^{\delta}\left(P_{T}-P_{m} \sin (\delta)\right) d \delta= \\
=P_{m}(1-\cos (\delta))-P_{T} \delta .
\end{gathered}
$$

Комбинируя выражение для потенциальной энергии (14) и уравнение для полной энергии (13), получаем выражение для сепаратрисы

$$
\omega(\delta)=\sqrt{\frac{2 k}{T_{J}}\left[P_{T}\left(\delta-\delta_{\text {кр }}\right)+P_{m}\left(\cos (\delta)-\cos \left(\delta_{\text {кр }}\right)\right)\right]},
$$

$k$ - коэффициент преобразования радианов в градусы.

Используя выражение для сепаратрисы (15), строим потенциальную кривую и фазовый портрет, которые показывают очевидную классификацию точек положения равновесия (рис. 3). Cenapaтриса разделяет фазовую плоскость на устойчивые и неустойчивые решения.

Очевидно, что устойчивые решения [19], замкнутые кривые внутри сепаратрисы, близкие к центру, будут напоминать колебания угла $\delta(t)$ близкие к гармоническим колебаниям. Решения за сепаратрисой неустойчивые, начинаются и заканчиваются большими значениями угла. В этом случае энергия возмущения, пришедшая из сети в генератор, значительно превосходит энергию генератора. Такие значения угла являются опасными для системы и приводят к аварийным ситуациям.

И наконец, пограничные решения, находящиеся на сепаратрисе, - это солитонные решения, peшения со всплеском амплитуды.

Чтобы рассмотреть возможные решения нелинейного уравнения, будем использовать численный метод решения системы уравнений (2) методом Рунге-Кутта 4-го порядка [20-22]. Приведем примеры определения качания угла генератора для одномашинной системы при трех различных случаях (рис. 4). 


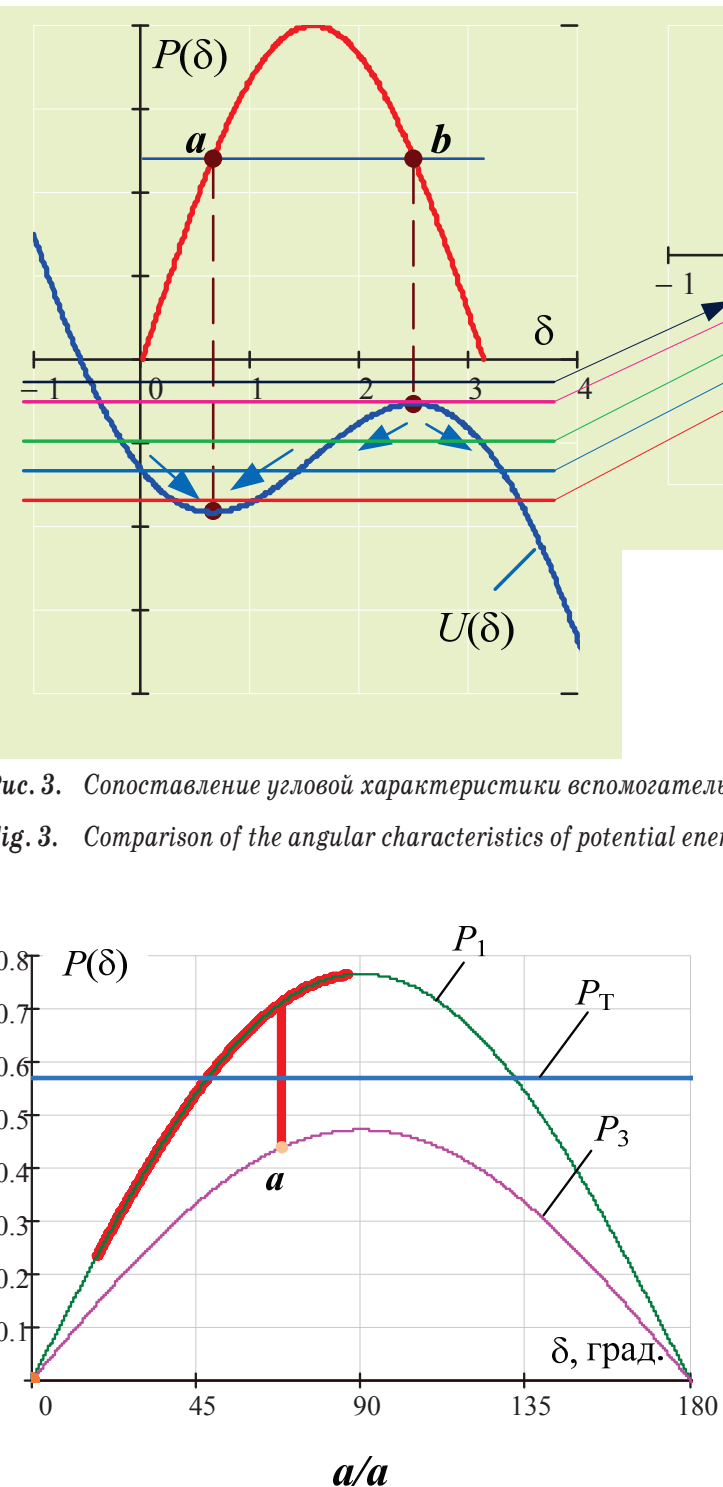

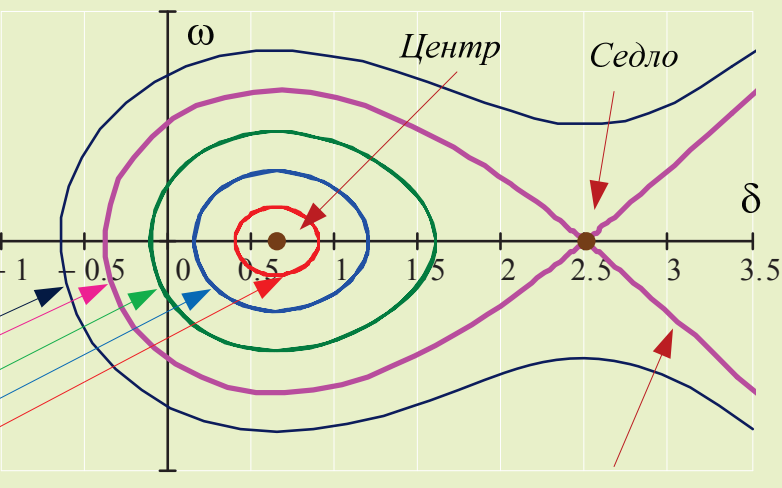

Cenapampuca

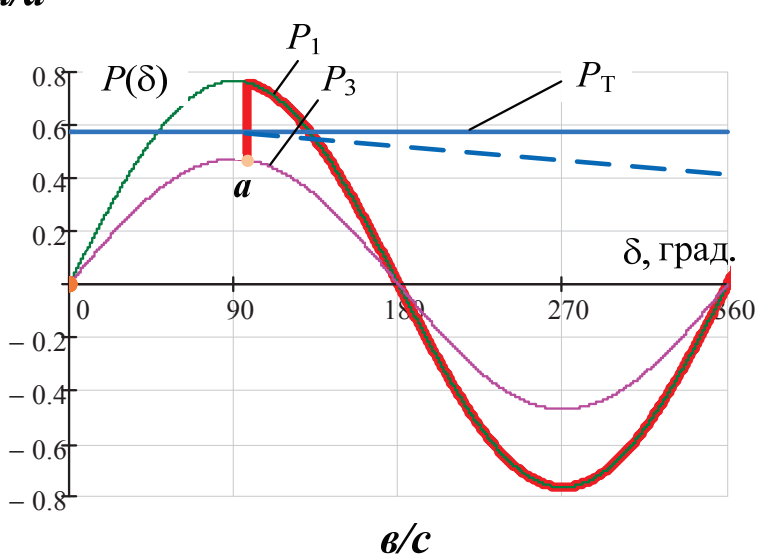

Pис. 4. Восстановление после возмущения в точке а: а) не приводит к нарушению устойчивости работы генератора; б) приводит си стелу на границу диналической устойчивости; в) приводит к нарушению диналической устойчивости, после чего происходит уленьшение мощности турбины с последующил восстановлением синхронизла

Fig. 4. Recovery after disturbance at point a: a) does not violate generator stability; $b$ ) brings the system to the border of dynamic stability; c) leads to violation of dynamic stability, after which there is a decrease in turbine power with subsequent restoration of synchronism 

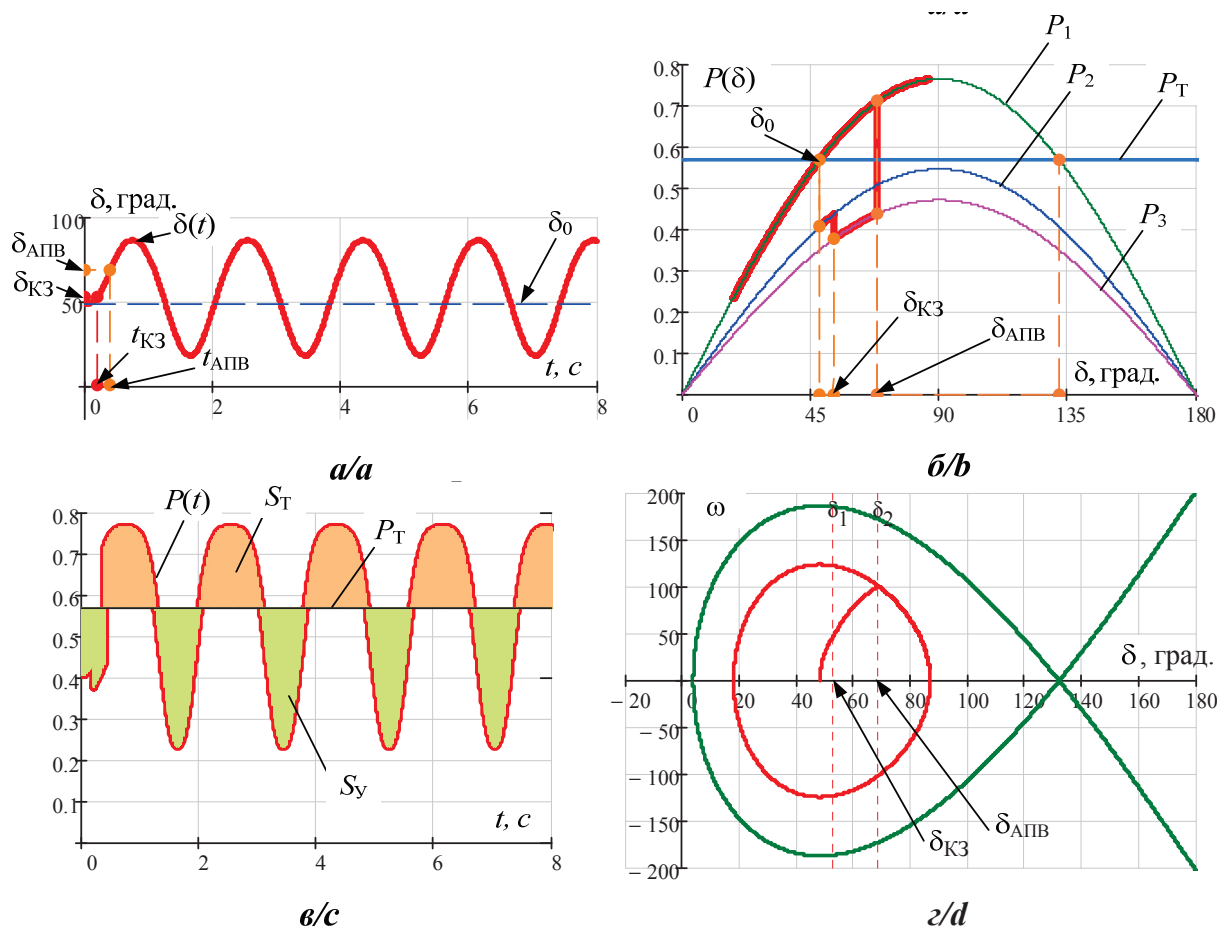

Pис. 5. Прилер устойчивого решения: а) колебания угла в окрестности точки положения равновесия; б) график изленения активной мощности после удачного срабатывания автоматического повторного включения; в) изменение активной мощности во времени; г) фазовый портрет динамической системы, решение находятся в пределах сепаратрисы

Fig. 5. Example of a stable solution: a) angle oscillations in the vicinity of the equilibrium point; $b$ ) graph of the change in active power after a successful automatic reclosing; $c$ ) change in active power over time; $d$ ) phase portrait of a dynamic system, the solution is located within the limits of the separatrix
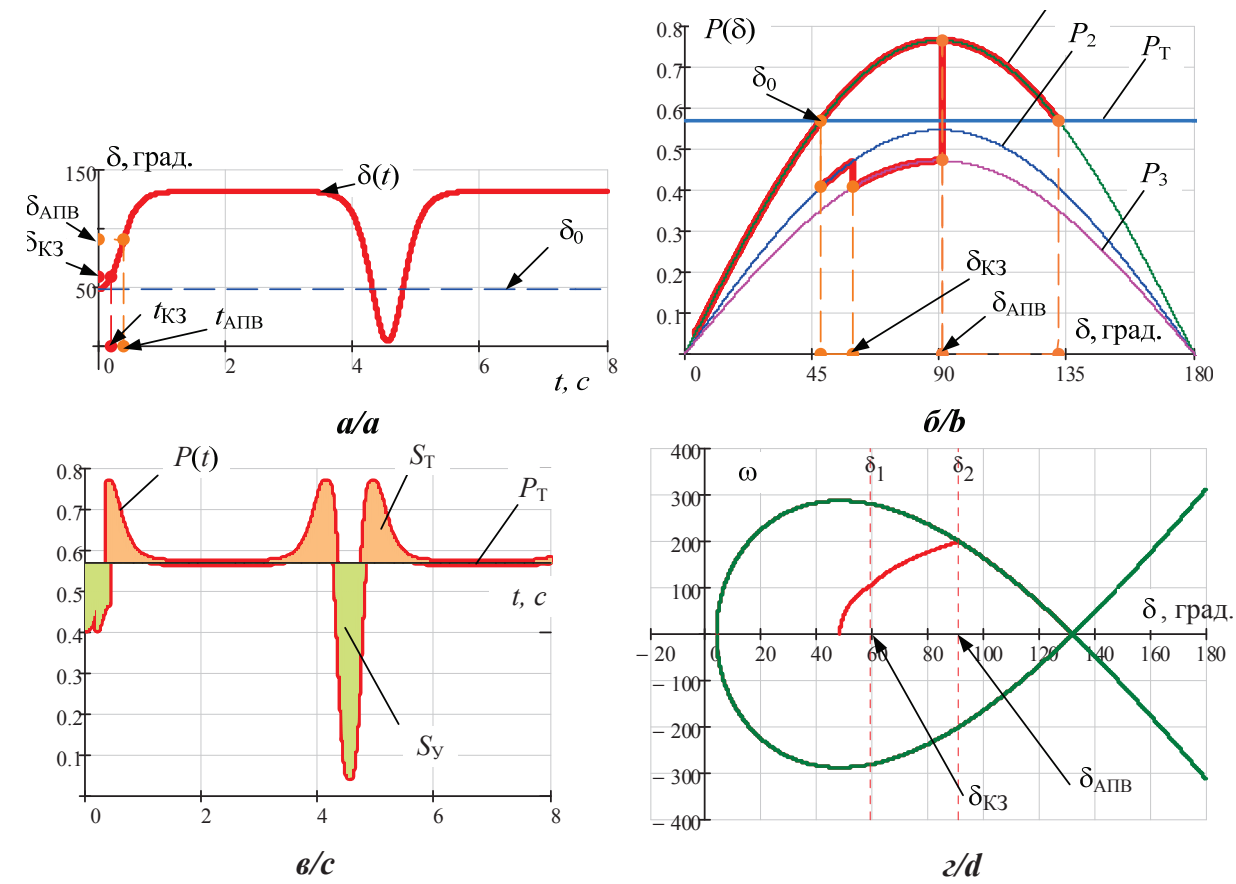

Pис.6. Прилер, когда решение находится на границе устойчивости: а) колебания угла в окрестности точки положения равновесия имеет солитонное решение; б) график изменения активной мощности после запоздалого срабатывания автоматического повторного включения; в) изменение активной мощности во времени; г) фазовый портрет динамической системы, решение находятся на сепаратрисе

Fig. 6. Example when the solution is on the stability boundary: a) angle oscillations around the equilibrium point has a soliton solution; б) graph of the change in active power after a delayed automatic reclosing; c) change in active power over time; d) phase portrait dynamical system, the solution is on a separatrix 


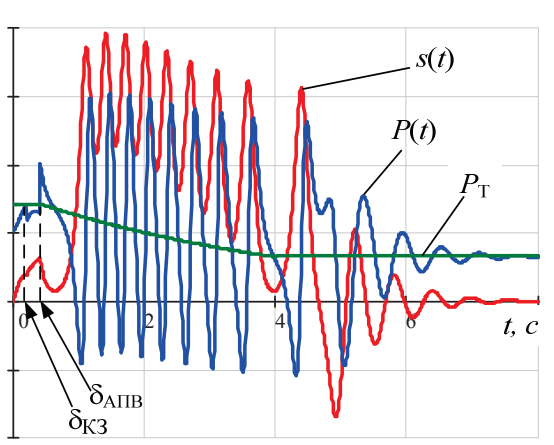

$a / a$

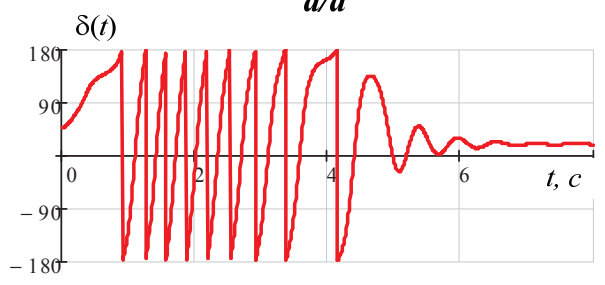

$\sigma / b$

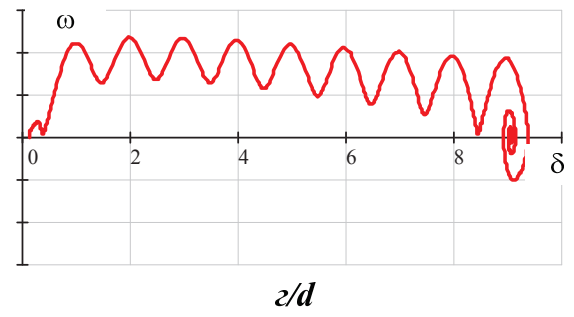

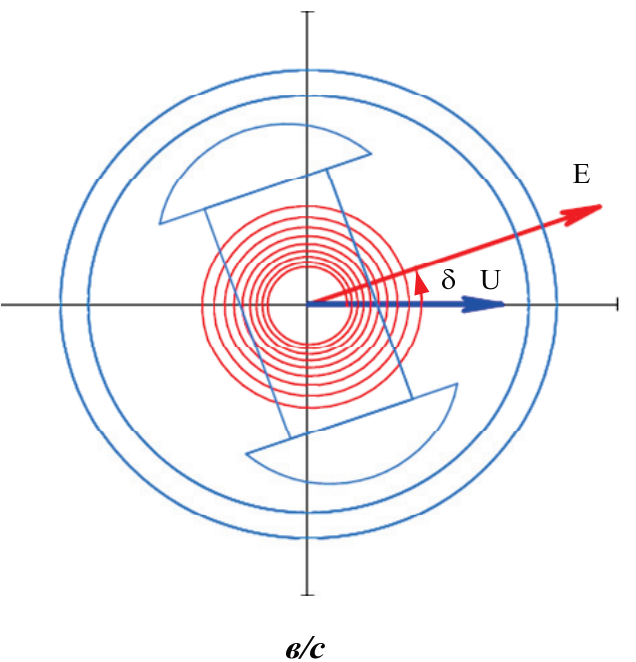

Рис.7. Пример ресинхронизации, когда происходит нарушение устойчивости с последующим его восстановлением: а) график изменения активной мощности и мощности турбины во времени, изменение скольжения ( производной угла во времени); б) график изменения угла качания генератора во времени; в) число оборотов генератора при нарушении синхронного режима; г) фазовый портрет динамической системы, решение находятся за сепаратрисой и на сепаратрисе

Fig. 7. Example of resynchronization, when there is a violation of stability followed by its restoration: a) graph of the change in active power and power of the turbine over time, change in the slip (derivative of the angle over time); $b$ ) graph of the change in generator oscillation angle over time; c) generator revolutions number in case of synchronous violation; d) phase portrait of the dynamic system, the solution is behind the separatrix and on the separatrix

1. Возмущение системы не приводит к нарушению устойчивости работы генератора.

2. Возмущение системы приводит систему на границу динамической устойчивости.

3. Возмущение системы приводит к нарушению динамической устойчивости, после чего происходит уменьшение мощности турбины с последующим восстановлением синхронизма.

В приведённых примерах на рис. 5-7 рассчитывается возмущение: на линии связи происходит однофазное короткое замыкание, которое ликвидируется отключением поврежденной фазы. С некоторой выдержкой времени происходит успешное повторное включение этой фазы, и схема возвращается в исходное состояние: $P_{1}-$ исходный, нормальный режим работы генератора; $P_{2}$ - аварийный режим, режим однофазного короткого замыкания, который ликвидируется отключением поврежденной фазы, далее происходит неполнофазный режим $-P_{3}$.

\section{СПИСОК ЛИТЕРАТУРЫ}

1. Baerthlein W., Audring D. Creating optimal power supply for extensive onshore oil fields // International Petroleum Exhibition and Conference. - Abu Dhabi,, 2018. - P. 49-53.

2. Букреев В.Г., Шандарова Е.Б., Рулевский В.М. Многомерная модель системы электропитания погружного технологического оборудования // Известия Томского политехнического университета. Инжиниринг георесурсов. - 2018. - Т. 329. - № 4. C. $119-131$.

\section{Заключение}

Рассмотрены возможные варианты динамической устойчивости работы генератора при коротких замыканиях. Обнаружено, что при приближении к границе динамической устойчивости возникают солитоны - одиночные волны угла генератора. При распространении эти волны ведут себя как частицы, что позволяет производить анализ обмена энергиями (потоками мощности) как обмен механических частиц энергиями. При нарушении устойчивости возникают гармонические колебания, которые преобразуются в группу солитонов (рис. 6). Распространение солитонов можно рассматривать как распространение частиц. В частности, известно, что солитоны с большей амплитудой будут обгонять солитоны с меньшей амплитудой, позволяя представить картину перетоков мощности. Чтобы привести систему в синхронный режим, необходимо уменьшать активную мощность турбины, добиваясь равновесия между генерацией и потреблением активной мощности.

3. Isaev Y.N., Kolchanova V.A., Tarasenko S.S., Tikhomirova O.V. Mathematical models of synchronous generators for different spatial distances of disturbance point // Proceedings of 2015 International Conference on Mechanical Engineering, Automation and Control Systems. - Tomsk, 2015. - № 124 (1). - P. 1-5.

4. Belyaev A.N., Bryantsev A.M., Smolovik S.V. Magnetically controlled shunt reactor operation experience in $110-500 \mathrm{kV}$ power grids // CIGRE Session 46. - Stara Lesna, Slovakia, 2016. P. 142-146. 
5. Инженерные основы новой энергетики / А.Н. Власов, С.В. Галкин, Ю.И. Гребенченко, О.В. Ольшанский, 0.0. Тужиков. Волгоград: Принт, 2008. - 336 с.

6. Brown M.P., Smith P.W. High power pulsed soliton generation at radio and microwave frequencies // Proc. $11^{\text {th }}$ IEEE Int. Pulsed Power Conf. - Baltimore, 1997. - P. 346-354.

7. Hernández S., Pontón M., Suárez A. Simulation method for complex multivalued curves in injection-locked oscillators // IEEE Trans. Microw. Theory Techn. - 2017. - V. 65 (11). P. 4046-4062

8. Хорошавин В.С., Зотов А.В., Мокрушин С.А. Общий подход к представлению динамики процесса в пространстве состояний // Advanced Science. - 2017. - № 2. - C. 38-46.

9. Mathematical simulation application for research of nonuniform distributed-parameter circuit transients / E.0. Kuleshova, A.A. Plyusnin, E.B. Shandarova, 0.V. Tikhomirova // IOP Conference Series: Materials Science and Engineering. - 2016 - V. 124 (1). P. $1-5$.

10. Rowlands G., Rozmej P., Infeld E. Single soliton solution to the extended KdV equation over uneven depth // The European Physical Journal E. - 2017. - V. 40 (11). - P. 1-5.

11. Инфельд Э., Роуландс Дж. Нелинейные волны, солитоны и хаос. - М.: Физматлит, 2006. - 480 с.

12. Nikoo M.S., Hashemi S.M.-A.. New soliton solution of a varactorloaded nonlinear transmission line // IEEE Trans. Microw. Theory Tech. - 2017. - V. 65 (11). - P. 4084-4092.

13. Лэм Дж. Л. Введение в теорию солитонов. - М.: Мир, 1983. $294 \mathrm{c}$.

14. Ахмедиев Н.Н., Анкевич А. Солитоны. Нелинейные импульсы и пучки. - М.: Физматлит, 2003. - 304 с.

15. An efficient analysis for N-soliton, Lump and Lump - Kink solutions of time-fractional (2+1)-Kadomtsev-Petviashvili equation M. Hamid, M. Usman, T. Zubair, R.U. Haq, A. Shafee // Physica
A: Statistical Mechanics and its Applications. - 2019. - V. 528. № 121320. - P. 1-13.

16. Mabrouk S.M., Rashed A S. N-solitons, Kink and periodic wave solutions for (3+1)-dimensional Hirota bilinear equation using three distinct techniques // Chinese Journal of Physics. - 2019. V. 60. - P. 48-60.

17. Melnikov L. Soliton fission and fusion in dispersion oscillating fiber and correlation properties of the pulses // Proc. 2016 International Conference Laser 0ptics. - St. Petersburg, 2016. V. R87. - P. 1-5.

18. Karczewska A., Rozmej P., Infeld E. Shallow-water soliton dynamics beyond the korteweg-de vries equation // Physical Review E - Statistical, Nonlinear, and Soft Matter Physics. - 2014. V. 90 (1). - P. 1-5.

19. Goldstein P., Infeld E. Stability of ion acoustic nonlinear waves and solitons in magnetized plasmas // Journal of Plasma Physics. - 2016. - V. 82 (6). - P. 1-5.

20. Assessment of thermodynamic parameters of plasma shock wave / O.V. Vasileva, Y.N. Isaev, A.A. Budko, A.I. Filkov // Journal of Physics: Conference Series. - 2014. - V. 552 (1). - P. 1-5.

21. Kolchanova V.A. Computational modeling of the tesla coil parameters // Procю of the $8^{\text {th }}$ International Scientific and Practical Conference of Students, Post-Graduates and Young Scientists: Modern Technique and Technologies, MTT. - Tомск, 2002. № 1213709. - P. 32-33.

22. Дискретная математическая модель на основе билинейного преобразования синхронной электрической машины с витковым замыканием в обмотке ротора В.И. Полищук, В.В. Тимошкин, А.С. Глазырин, Е.В. Боловин // Электротехника. 2019. - № 2. - C. 24-31.

Поступила 13.12.2019 2.

\section{Информация об авторах}

Исаев Ю.Н., доктор физико-математических наук, профессор отделения электроэнергетики и электротехники Инженерной школы энергетики Национального исследовательского Томского политехнического университета.

Колчанова B.A., кандидат технических наук, доцент отделения электроэнергетики и электротехники Инженерной школы энергетики Национального исследовательского Томского политехнического университета.

Kулешова E.O., кандидат физико-математических наук, доцент отделения электроэнергетики и электротехники Инженерной школы энергетики Национального исследовательского Томского политехнического университета.

Филипас A.A., кандидат технических наук, доцент отделения автоматизации и робототехники Инженерной школы информационных технологий и робототехники Национального исследовательского Томского политехнического университета. 


\title{
SOLITON SOLUTION OF ELECTROMECHANICAL TRANSIENT PROCESSES IN ELECTRIC POWER SYSTEMS
}

\author{
Yusup N. Isaev', \\ isaev_yusup@mail.ru \\ Veroniaka A. Kolchanova', \\ ni105@mail.ru \\ Elena 0. Kuleshova', \\ kul_2004@mail.ru \\ Alexander A. Filipas', \\ filipas@tpu.ru \\ 1 National Research Tomsk Polytechnic University, \\ 30, Lenin Avenue, Tomsk, 634050, Russia.
}

The paper introduces the solution of the wave equation of the electromechanical system of electric power engineering in the form of a soliton - solitary wave. The causes of such waves are described. The solutions are considered in the phase plane, a numerical example of the soliton solution was given.

The main aim of the research is to find the soliton solutions in transients of electromechanical systems, and explain the causes of their occurrence, explain this physical phenomenon, determine the role this phenomenon plays in the sustainability assessment, and propose the measures to eliminate the violation of stability in the presence of a soliton wave.

Methods: phase plane method, numerical differential equation by the Runge-Kutt method, state space method.

Results. It was found out that when the turbine-generator equation solution is approaching to the dynamic stability boundary, the solitons - solitary waves of the generator angle - appear.

Conclusions. These waves behave like particles at propagation, that allows analyzing the exchange of energies (power flows) as the exchange in mechanical particles with energies. When stability is violated, harmonic oscillations arise, which are transformed into a group of solitons which propagation can be considered as the propagation of particles.

\section{Key words:}

Wave surge, soliton, electromechanical transients, dynamic system, phase space.

\section{REFERENCES}

1. Baerthlein W., Audring D. Creating optimal power supply for extensive onshore oil fields. International Petroleum Exhibition and Conference. Abu Dhabi, 2018. pp. 49-53.

2. Bukreev V.G., Shandarova E.B., Rulevskiy V.M. Power supply system model of remote processing equipment. Bulletin of the Tomsk Polytechnic University, Geo Assets Engineering, 2018, vol. 329, no. 4, pp. 119-131. In Rus.

3. Isaev Y.N., Kolchanova V.A., Tarasenko S.S., Tikhomirova 0.V. Mathematical models of synchronous generators for different spatial distances of disturbance point. Proc. of 2015 International Conference on Mechanical Engineering, Automation and Control Systems. Tomsk, 2015. Vol. 124, no. 1, pp. 1-5.

4. Belyaev A.N., Bryantsev A.M., Smolovik S.V. Magnetically controlled shunt reactor operation experience in $110-500 \mathrm{kV}$ power grids. CIGRE Session 46. Stara Lesna; Slovakia, 2016. pp. 142-146.

5. Vlasov A.N., Galkin S.V., Grebenchenko Yu.I., Olshansky 0.V., Tuzhikov 0.0. Inzhenernye osnovy novoy energetiki [Engineering basics of new energy]. Volgograd, Print Publ., 2008. 336 p.

6. Brown M.P., Smith P.W. High power pulsed soliton generation at radio and microwave frequencies. Proc. $11^{\text {th }}$ IEEE Int. Pulsed Power Conf. Baltimore, 1997. pp. 346-354.

7. Hernández S., Pontón M., Suárez A. Simulation method for complex multivalued curves in injection-locked oscillators. IEEE Trans. Microw. Theory Techn., 2017, vol. 65, no. 11, pp. 4046-4062.

8. Khoroshavin V.S., Zotov A.V., Mokrushin S.A. General approach to the representation of the dynamics of the process in the state space. Advanced Science, 2017, no. 2, pp. 38-46. In Rus.
9. Kuleshova E.O., Plyusnin A.A., Shandarova E.B., Tikhomirova O.V. Mathematical simulation application for research of nonuniform distributed-parameter circuit transients. IOP Conference Series: $M a$ terials Science and Engineering, 2016, vol. 124, no 1, pp. 1-5.

10. Rowlands G., Rozmej P., Infeld E. Single soliton solution to the extended KdV equation over uneven depth. The European Physical Journal E, 2017, vol. 40, no. 11, pp. 1-5.

11. Infeld E., Roulands J. Nelineynye volny, solitony $i$ khaos [Nonlinear waves, solitons and chaos]. Moscow, Fizmatlit Publ., 2006. $480 \mathrm{p}$.

12. Nikoo M.S., Hashemi S.M.-A. New soliton solution of a varactorloaded nonlinear transmission line. IEEE Trans. Microw. Theory Tech., 2017, vol. 65, no. 11, pp. 4084-4092.

13. Lam J.L. Vvedenie $v$ teoriyu solitonov [Introduction to the theory of solitons]. Moscow, Mir Publ., 2006. 294 p.

14. Akhmediev N.N., Ankevich A. Nelineynye impulsy i puchki [Solitons. Nonlinear pulses and beams]. Moscow, Fizmatlit Publ., 2003. $304 \mathrm{p}$.

15. Hamid M., Usman M., Zubair T., Haq R.U., Shafee A. An efficient analysis for N-soliton, Lump and Lump-Kink solutions of time-fractional (2+1)-Kadomtsev-Petviashvili equation. Physica A: Statistical Mechanics and its Applications, 2019, vol. 528, pp. 1-13.

16. Mabrouk S.M., Rashed A.S. N-solitons, Kink and periodic wave solutions for (3+1)-dimensional Hirota bilinear equation using three distinct techniques. Chinese Journal of Physics, 2019, vol. 60 , pp. 48-60.

17. Melnikov L. Soliton fission and fusion in dispersio n oscillating fiber and correlation properties of the pulses. Proc. 2016 Interna- 
tional Conference Laser Optics. St. Petersburg, 2016. Vol. R87, pp. 1-5.

18. Karczewska A., Rozmej P., Infeld E. Shallow-water soliton dynamics beyond the korteweg-de vries equation. Physical Review E Statistical, Nonlinear, and Soft Matter Physics, 2014, vol. 9, no. 1, pp. 1-5.

19. Goldstein P., Infeld E. Stability of ion acoustic nonlinear waves and solitons in magnetized plasmas. Journal of Plasma Physics, 2016, vol. 82, no. 6, pp. 1-5.

20. Vasileva O.V., Isaev Y.N., Budko A.A., Filkov A.I. Assessment of thermodynamic parameters of plasma shock wave. Journal of Physics: Conference Series, 2014, vol. 552, no. 1, pp. 1-5.
21. Kolchanova V.A. Computational modeling of the tesla coil parameters. Proc. of the $8^{\text {th }}$ International Scientific and Practical Conference of Students, Post-Graduates and Young Scientists: Modern Technique and Technologies, MTT. Tomsk, 2002. Vol. 1213709, pp. 32-33.

22. Polishchuk V.I., Timoshkin V.V., Glazyrin A.S., Bolovin E.V. A discreet mathematical model based on the bilinear transformation of a synchronous electric machine with a turn-to-turn fault in the rotor winding. Russian Electrical Engineering, 2019, vol. 90 (2), pp. 113-120.

Received: 13 December 2019.

\section{Information about the authors}

Yusup N. Isaev, Dr. Sc., professor, National Research Tomsk Polytechnic University.

Veroniaka A. Kolchanova, Cand. Sc., associate professor, National Research Tomsk Polytechnic University.

Elena O. Kuleshova, Cand. Sc., associate professor, National Research Tomsk Polytechnic University.

Alexander A. Filipas, Cand. Sc., associate professor, National Research Tomsk Polytechnic University. 